\title{
Breathlessness and Restrictive Lung Disease: An Important Diabetes-Related Feature in Patients with Type 2 Diabetes
}

\author{
Stefan Kopf ${ }^{a, b}$ Jan B. Groener ${ }^{a, b}$ Zoltan Kender ${ }^{a, b}$ Thomas Fleming ${ }^{a, b}$ \\ Maik Brune $^{a}$ Christin Riedinger ${ }^{a}$ Nadine Volk ${ }^{c, d}$ Esther Herpel ${ }^{c, d}$ \\ Dominik Pesta ${ }^{e}, f$ Julia Szendrödie, f, g Mark O. Wielpütz ${ }^{\text {h, } i}$ \\ Hans-Ulrich Kauczor $^{\text {h, }}$ Hugo A. Katus $^{j}$ Michael Kreuter ${ }^{i, k}$ Peter P. Nawroth ${ }^{\text {a, b, I }}$ \\ ${ }^{a}$ Department of Endocrinology, Diabetology, and Clinical Chemistry, Internal Medicine I, University Hospital of

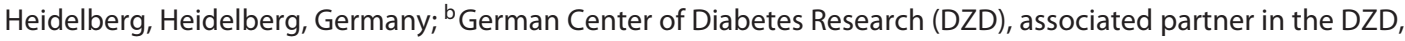 \\ München-Neuherberg, Germany; ${ }^{C}$ Institute of Pathology, University of Heidelberg, Heidelberg, Germany; ${ }^{\mathrm{d}}$ Tissue

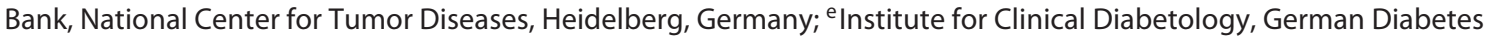 \\ Center, Düsseldorf, Germany; ${ }^{f}$ German Center for Diabetes Research (DZD), partner in the DZD, München-Neuherberg, \\ Germany; ${ }^{9}$ Department of Endocrinology and Diabetology, Medical Faculty, Heinrich Heine University, Düsseldorf,

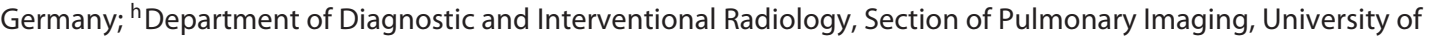

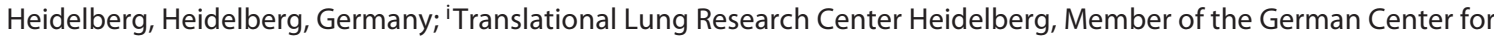 \\ Lung Research, Heidelberg, Germany; ${ }^{j}$ Department of Cardiology, Angiology, and Pneumology, Internal Medicine III, \\ University Hospital of Heidelberg, Heidelberg, Germany; ${ }^{k}$ Center for Interstitial and Rare Lung Diseases, Pneumology, \\ Thoraxklinik, University of Heidelberg, Heidelberg, Germany; 'Joint-IDC, Institute for Diabetes and Cancer at Helmholtz \\ Zentrum Munich and University of Heidelberg, Heidelberg, Germany
}

\section{Keywords}

Fibrosing interstitial lung disease · Type 2 diabetes .

Albuminuria $\cdot$ Diabetes-related complications

\begin{abstract}
Background: Diabetes mellitus is a significant comorbidity of interstitial lung disease (ILD). Objectives: The aim of this study was to investigate the incidence of restrictive lung disease (RLD) and ILD in patients with prediabetes and type 2 diabetes (T2D). Methods: Forty-eight nondiabetics, 68 patients with prediabetes, 29 newly diagnosed T2D, and 110 patients with long-term T2D were examined for metabolic control, diabetes-related complications, breathlessness, and lung function. Five participants with T2D, breathlessness,
\end{abstract}

and RLD underwent multidetector computed tomography (MDCT) and a Six-Minute Walk Test (6MWT). Lung tissue from 4 patients without diabetes and from 3 patients with T2D was histologically examined for presence of pulmonary fibrosis. Results: Breathlessness in combination with RLD was significantly increased in patients with prediabetes and T2D $(p<0.01)$. RLD was found in $9 \%$ of patients with prediabetes, in $20 \%$ of patients with newly diagnosed T2D, and in $27 \%$ of patients with long-term T2D. Thus, patients with long-term T2D had an increased risk of RLD (OR 5.82 [95\% CI 1.71-20.5], $p<0.01)$. RLD was significantly associated with glucose metabolism and albuminuria ( $p<0.01)$; furthermore, presence

Clinical Trial Registration: NCT03022721.

\section{KARGER}

(c) 2018 S. Karger AG, Basel

E-Mail karger@karger.com

www.karger.com/res
Stefan Kopf, MD

Department of Endocrinology, Diabetology, and Clinical Chemistry, Internal Medicine I University Hospital of Heidelberg, Im Neuenheimer Feld 410 DE-69120 Heidelberg (Germany)

E-Mail stefan.kopf@med.uni-heidelberg.de 
of nephropathy increased the risk of RLD (OR 8.57 [95\% Cl 3.4-21.9], $p<0.01)$ compared to nondiabetics. MDCT revealed ILD in 4 patients, the 6MWT correlated with the extent of ILD, and histological analysis showed fibrosing ILD in patients with T2D. Conclusions: This study demonstrates increased breathlessness and a high prevalence of RLD in patients with T2D, indicating an association between diabetes and fibrosing ILD.

(c) 2018 S. Karger AG, Basel

\section{Introduction}

Breathlessness is reported by $25 \%$ of patients in the general daily ambulatory setting [1]. While coronary heart disease or chronic heart failure is the reason for dyspnea in approximately $11 \%$ of these cases, one may speculate that acute and chronic pulmonary diseases may be amongst the main causes for breathlessness $[1,2]$. In addition to chronic obstructive pulmonary disease, which is mainly caused by smoking, restrictive lung diseases (RLDs), such as fibrosing interstitial lung disease (ILD), have an incidence rate of $19.36-34.34 \%$ per 100,000 patient-years, while nonspecific fibrosis showed the highest frequency with $7.5 \%$ in a population-based study in Denmark [3]. Fibrosing ILDs may be secondary to other diseases (i.e., connective tissue diseases, drugs, or inhalative antigens as in hypersensitivity pneumonitis) $[4,5]$. However, pulmonary fibrosis can also appear as an idiopathic form, mainly as idiopathic pulmonary fibrosis (IPF). In IPF there is continuous epithelial microinjury with activation of inflammatory mediators leading to fibrosis [6]. Since similar injuries are also observed in diabetes-related complications, it has been discussed whether fibrosing ILD is a possible diabetes-related complication [7]. Also, IPF patients with diabetes have a worse prognosis compared to IPF patients without diabetes [8]. However, only few studies have investigated lung function in patients with diabetes, describing a decrease in pulmonary function in patients with diabetes without more precise differentiation [9-11]. On the other side, diabetes seems to be an important comorbidity and might be a risk factor for the development of IPF aggravation [12-14]. Yet, no study exists with a focus on breathlessness as a consequence of specific pulmonary dysfunction in patients with diabetes and its relation to diabetic complications.

Thus, the aim of this study was to investigate breathlessness, RLD, and fibrosing ILD in patients with prediabetes and type 2 diabetes (T2D) compared to nondiabet- ics, with the hypothesis that patients with T2D and late diabetic complications have an increased risk of RLD and pulmonary fibrosis.

\section{Subjects and Methods}

\section{Study Design and Methods}

Over 280 participants were screened from May 5, 2015 until January 27, 2017 at the outpatient clinic of the University Hospital of Heidelberg. The main inclusion criteria were patients with or without T2D or prediabetes aged 18-75 years. Participants responded to a study call of the University Hospital of Heidelberg via Internet or advertisements. An oral glucose tolerance test with 75 g glucose was performed in participants without known T2D. Prediabetes was defined as fasting glucose levels of $100-125 \mathrm{md} /$ dL or a 2-h glucose level of 140-199 mg/dL after 75 g glucose intake. Participants with glucose levels below these limits were defined as normal (healthy controls). Participants with glucose levels above the limit and absence of GAD or IA2 antibodies were defined as newly diagnosed T2D. Exclusion criteria included type 1 diabetes, end-stage heart, liver, or kidney failure, known glomerulonephritis or glomerulopathy of another cause, malignant diseases, chronic alcohol or illegal drug abuse, current nicotine abuse, known lung diseases including chronic obstructive pulmonary disease, pulmonary hypertension, and obstructive sleep apnea. For all laboratory values shown, blood was drawn in the morning under fasting conditions and immediately processed in the central laboratory of the University Hospital of Heidelberg under standardized conditions. The estimation of glomerular filtration rate was calculated using the CKD-EPI formula [15].

\section{Breathlessness, Lung Function Testing, and Peripheral Oxygen} Saturation

All participants were screened for breathlessness using the modified Medical Research Council (mMRC) score [16]. Participants were graded from 0 to 4 depending on breathlessness-related activities reported. Spirometry, body plethysmography, and carbon monoxide-based diffusing capacity measurements were performed using the body plethysmograph PowerCube Body+ by Ganshorn Medizin Electronic GmbH (Niederlauer, Germany). Lung function testing was performed by specialized trained technicians according to the guidelines and reference values of the American Thoracic Society (ATS) and the European Respiratory Society (ERS) [17, 18]. Single-breath diffusing capacity of the lung for carbon monoxide $\left(\mathrm{SB}-\mathrm{DL}_{\mathrm{CO}}\right.$ ) was determined using a standard measurement gas containing $21 \%$ oxygen, $10 \%$ helium, and $0.3 \%$ carbon monoxide. All measured parameters were adjusted for age, sex, and body mass index (BMI). Diffusion measurements were adjusted to the current hemoglobin value. Forced vital capacity (FVC), forced expiratory volume in $1 \mathrm{~s}$ adjusted to vital capacity $\left(\mathrm{FEV}_{1} / \mathrm{VC}\right), \mathrm{SB}-\mathrm{DL}_{\mathrm{CO}}$, and total lung capacity measured with body plethysmography (TLC-B) were given in percent predicted and were used for analysis. Patients who showed reduced FVC $(<80 \%)$, TLC-B $(<80 \%)$, and $\mathrm{DL}_{\mathrm{CO}}(<80 \%)$ in presence of normal $\mathrm{FEV}_{1} / \mathrm{VC}(>70 \%)$ were categorized as having $\operatorname{RLD}[17,19,20]$. Peripheral oxygen saturation (in percent) was measured over $30 \mathrm{~s}$ via noninvasive pulse oximetry using the pulse oximeter PULOX PO-200 (Novidion GmbH, Cologne, Germany). This measure- 
ment was performed in a resting state after acclimatization to room temperature, and the highest value measured was used for analysis.

\section{Evaluation of Diabetes-Related Complications}

Urinary albumin and creatinine measurements were performed in morning spot urine under standardized conditions in the central laboratory of the University Hospital of Heidelberg. Urinary albumin-creatinine ratio was calculated in $\mathrm{mg} / \mathrm{g}$ and values $>30 \mathrm{mg} / \mathrm{g}$ were defined as pathological. All patients received a clinical examination procedure comprising Achilles tendon reflex, vibration perception using a Rydel-Seiffer graduated C64 to C128 tuning fork, pain sensation using a monofilament $(0.1 \mathrm{~N})$, and cold-warm discrimination using a Tip Therm ${ }^{\circledR}$, as described previously [21]. Regarding the neuropathy deficit score, a score $>2$ points was defined as distal sensomotoric polyneuropathy [22]. One-field retinal photography of both eyes was performed in all participants to detect diabetes-related retinopathy by using a NIDEK AFC-230 ${ }^{\circledR}$ (NIDEK Co. Ltd., Padova, Italy) combined with a Canon EOS 5D Mark II (Canon Deutschland GmbH, Krefeld, Germany). All photographs were analyzed by specially trained physicians.

\section{Chest Computed Tomography and Six-Minute Walk Test}

Participants with increased breathlessness, restrictive lung function, and inexplicable decreased $\mathrm{SB}-\mathrm{DL}_{\mathrm{CO}}<60 \%$ were asked to undergo multidetector computed tomography (MDCT) and a SixMinute Walk Test (6MWT) on the same day. Separate written informed consent was obtained for these procedures. Nonenhanced CT (iCT 256; Philips Medical Systems, Best, Netherlands) was acquired in full inspiratory breath-hold and supine position and reconstructed in a lung kernel with overlapping slices of $1.5 \mathrm{~mm}$ thickness. Two dedicated chest radiologists interpreted the images according to the ATS/ERS criteria for the diagnosis of pulmonary fibrosis $[23,24]$.

For the 6MWT, participants were instructed to walk a 300-meter corridor forward and backward at their own speed to get as much distance as possible within $6 \mathrm{~min}$ [25]. The distance was recorded by a distance measuring wheel (Rolson 50799; Rolson Tools Ltd., Reading, UK). Using the reference equation formula, the percent of predicted distance was related to the reached distance [25]. Dyspnea during the 6MWT was quantified using the modified Borg scale ranging from 0 to 10 [26].

\section{Staining of Lung Tissue}

Tissue samples were provided by the tissue bank of the National Center for Tumor Diseases (Heidelberg, Germany) in accordance with the regulations of the tissue bank and the approval of the local ethics committee. The tissue had not been obtained from patients studied in the cohort mentioned above. Lung tissue had been obtained in the course of surgical operations due to suspicion of tumor growth. Nonmalignant tissue specimens from 3 patients with T2D and 4 patients without diabetes were used for analysis. Tissue specimen of formalin-fixed and paraffin-embedded material $(3 \mu \mathrm{m})$ were cut using an HM 340E Rotary Microtome (Thermo Fisher Scientific) and stained with hematoxylin and eosin and (Masson's) trichrome. Images were acquired at $20 \times$ using a Hamamatsu NanoZoomer Digital Pathology (Herrsching am Ammersee, Germany) system.

Breathlessness and Restrictive Lung Disease

\section{Statistical Analyses}

For comparison between the different groups, one-way ANOVA was performed for metric variables and the $\chi^{2}$ test for dichotomous variables. Correlation analyses were performed using the Pearson correlation coefficient. Linear regression analyses were performed forward and backward to analyze independent associations between FVC, SB-DL $\mathrm{CO}_{\text {, }}$ and TLC-B with glucose metabolism (fasting glucose and $\mathrm{HbAlc}$ ). In these analyses, age, sex, BMI, patient group, history of smoking, known cardiovascular disease (CVD), and arterial hypertension were used as covariables.

Furthermore, logistic regression analyses were performed forward and backward with the likelihood ratio to calculate OR (Exp [B]) for RLD. Age, sex, BMI, history of smoking, CVD, and arterial hypertension were set as fixed covariables. For determination of further independent variables in these analyses, univariate analyses for each variable were performed to compare within the group of normal lung function and RLD. Variables with a $p$ value $<0.1$ were included in the regression models. For logistic regression analysis in the whole patient cohort, the following independent variables were included: age, sex, patient group, history of smoking, CVD, arterial hypertension, BMI, cholesterol, and triglycerides. Due to the continuously increasing $\mathrm{HbAlc}$ in the respective patient group, this variable was dispensed within the regression analysis in order to avoid a possible bias in the analysis. For logistic regression analysis in patients with T2D, the following independent variables were included: age, sex, history of smoking, CVD, arterial hypertension, BMI, HbAlc, presence of neuropathy, retinopathy, and nephropathy. Urinary albumin-creatinine ratio was log-transformed for this analysis to achieve normal distribution.

Statistical $p$ values $<0.05$ were defined as significant.

All analyses were performed using IBM SPSS 23.0 (IBM Corp., Armonk, NY, USA). Figures, graphs, and blots were visualized with IBM SPSS 23.0, GraphPad Prism 7.0 (GraphPad Software Inc., La Jolla, CA, USA), or NDP.view2 (Hamamatsu Photonics Deutschland $\mathrm{GmbH}$, Herrsching am Ammersee, Germany).

\section{Results}

Of 280 patients screened, 255 were included in this study comprising 48 nondiabetics (controls), 68 patients with proven prediabetes, 29 patients with newly diagnosed T2D, and 110 patients with long-term T2D. Twenty-five patients were excluded because of current nicotine abuse $(n=11)$, known chronic obstructive lung disease $(n=14)$, pulmonary hypertension $(n=5)$, or known chronic heart failure $(n=8)$. The patients with newly diagnosed T2D were significantly younger in comparison to the other participants, with a mean age of 51 years, while the participants with long-term T2D group were significantly older, with a mean age of 63 years (Table 1). More females were included in the control and the prediabetes group in comparison to the diabetes group $(p<0.05)$. More details are shown in Table 1. 
Table 1. Baseline characteristics

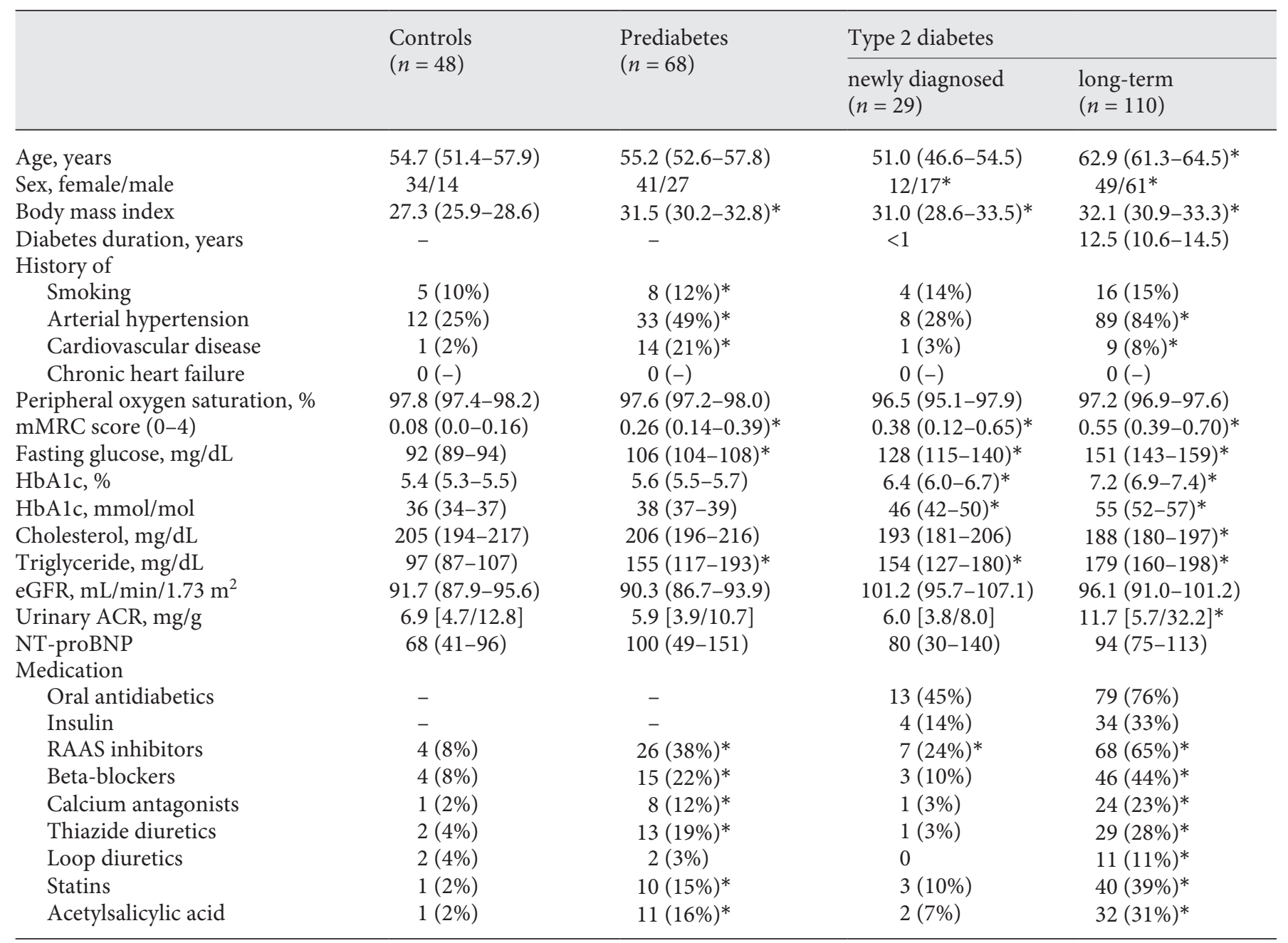

Data are given as mean $(95 \% \mathrm{CI}), n(\%)$, or median [25th/75th percentile]. ACR, albumin-creatinine ratio; eGFR, estimated glomerular filtration rate; mMRC, modified Medical Research Council; RAAS, renin-angiotensin-aldosterone system. ${ }^{\star}$ Significant difference $(p<0.05)$ compared to the control group.

None of the patients had a history of chronic heart failure, and NT-proBNP was within the normal range, without any significant differences between the groups. The mMRC breathlessness score showed significant differences between the groups $(F=6.2, p<0.01)$, with increased breathlessness in patients with long-term T2D compared to nondiabetics $(p<0.01)$ and to patients with prediabetes $(p<0.05)$ (Table 1$)$. RLD with reduced FVC, reduced $\mathrm{SB}-\mathrm{DL}_{\mathrm{CO}}$, and reduced TLC-B was more frequent in patients with long-term T2D (online suppl. Fig. S1; see www.karger.com/doi/10.1159/000488909 for all online suppl. material). One-way ANOVA showed a de- crease in FVC $(F=5.3, p<0.001), \mathrm{SB}_{-} \mathrm{DL}_{\mathrm{CO}}(F=5.1$, $p<0.001)$, and TLC-B $(F=8.4, p<0.001)$ depending on the patient group studied. None of the patients showed significant obstructive lung disorder as measured via $\mathrm{FEV}_{1} / \mathrm{VC}$. The mMRC score significantly increased in patients with RLD. One-way ANOVA showed decreases in $\operatorname{FVC}(F=19.5, p<0.001)$, SB-DL $\mathrm{CO}(F=53.4, p<0.01)$, and TLC-B $(F=26.4, p<0.01)$, and an increase in $\mathrm{FEV}_{1} /$ VC $(F=4.7, p<0.01)$, with increased breathlessness depending on different grades (mMRC score $0-4$; see online suppl. Fig. S2). None of the patients reported very severe breathlessness (mMRC score 4). 


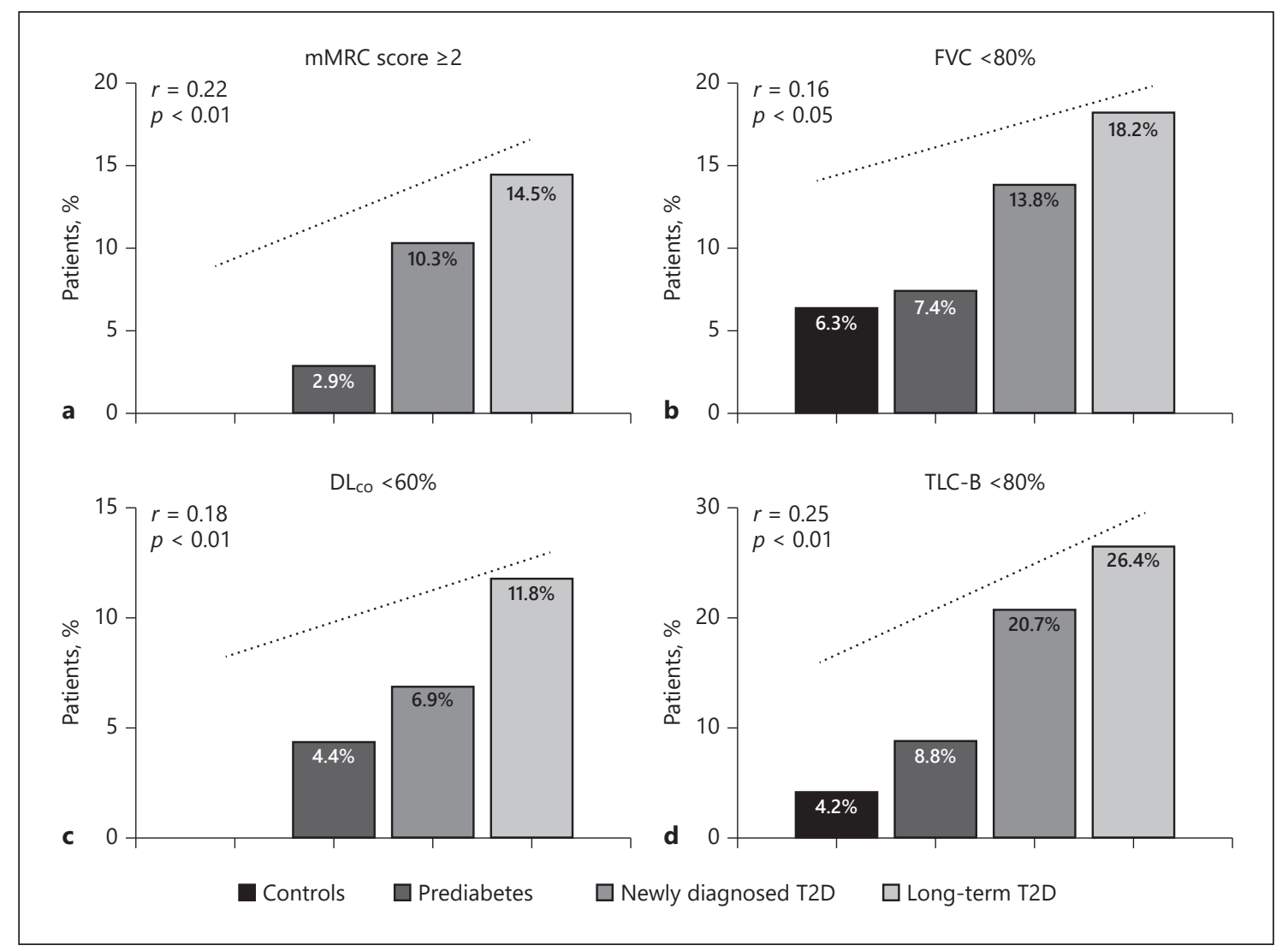

Fig. 1. Breathlessness and restrictive lung disease. Percentages of patients are shown in the bar graphs according

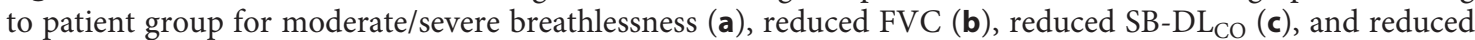
TLC-B (d). Additionally, results of linear-to-linear correlation analyses for each parameter with different patient groups are given as dotted lines together with correlation coefficients and $p$ values. FVC, forced vital capacity; mMRC, modified Medical Research Council; SB-DL ${ }_{C O}$, single-breath diffusing capacity of the lung for carbon monoxide; T2D, type 2 diabetes; TLC-B, total lung capacity measured with body plethysmography.

\section{Breathlessness and RLD}

Moderate (mMRC score 2) and severe breathlessness (mMRC score 3 ) was reported in 2.9\% $(n=2)$ of patients with prediabetes, in $10.3 \%(n=3)$ of patients with newly diagnosed T2D, and in $14.5 \%$ ( $n=12$ with moderate and $n=4$ with severe breathlessness) of patients with longterm T2D (Fig. 1a). Linear-to-linear correlation analysis showed a significant increase dependent upon patient group $(r=0.22, p<0.01)$. Mild breathlessness (mMRC score 1$)$ was reported by $4(8 \%)$ controls, by $14(21 \%)$ patients with prediabetes, by $5(17 \%)$ patients with newly diagnosed T2D, and by 24 (22\%) patients with long-term T2D (data not shown). Breathlessness was not significantly associated with history of smoking, arterial hypertension, or CVD. Furthermore, one-way ANOVA showed no significant association between $\mathrm{BMI}$ and different grades of breathlessness $(F=2.01, p=0.11)$.

With regards to RLD, reduced FVC $(<80 \%)$ was found in $18.2 \%$ of patients $(n=20)$ with long-term T2D and in $13.8 \%$ of patients $(n=4)$ with newly diagnosed T2D, whilst the number of patients with reduced FVC was lower in healthy controls $(6.3 \%, n=3)$ and patients with prediabetes $(7.4 \%, n=5)$ (Fig. 1b). Similar results were obtained for SB-DL $\mathrm{CO}$ (Fig. 1c) and TLC-B (Fig. 1d). Decreased SB-DL $\mathrm{CO}_{\mathrm{CO}}(<60 \%)$ was found in $6.9 \%$ of patients $(n=2)$ with newly diagnosed T2D and in $11.8 \%$ of patients $(n=13)$ with long-term T2D $(r=0.18, p<0.01)$ (Fig. 1c). Additionally, 20.7\% $(n=6)$ of patients with newly diagnosed T2D and $26.4 \%(n=29)$ of patients with long-term T2D had a decreased TLC-B $<80 \%$. Linear-to- 


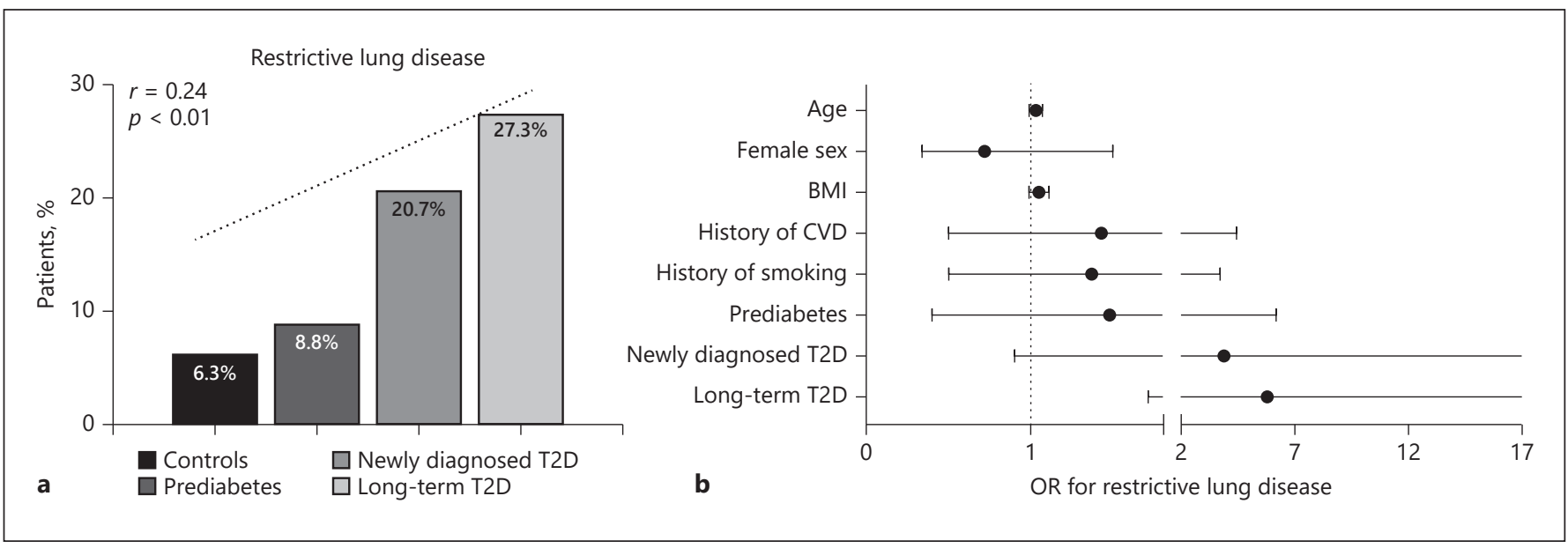

Fig. 2. Restrictive lung disease and independent risk factors. a Percentages of patients are shown in the bar graphs according to patient group for restrictive lung disease. Results of linear-to-linear correlation analyses are given as a dotted line together with correlation coefficient and $p$ value. $\mathbf{b}$ Calculated ORs with $95 \%$ CIs for restrictive lung disease plotted on the $x$ axis for selected clinical parameters; details are given in online supplementary Table S1. CVD, cardiovascular disease; T2D, type 2 diabetes.

linear correlation analysis showed a significant positive association $(r=0.25, p<0.05)$ (Fig. 1d).

Thus, patients with RLD were significantly more frequent in the T2D group: $27.3 \%$ of patients $(n=30)$ with long-term and $20.7 \%$ of patients $(n=6)$ with newly diagnosed T2D (Fig. 2a). Patients with RLD were less frequent in healthy controls and in patients with prediabetes. Linear-to-linear correlation analysis showed a significant positive association of RLD with diabetes $(r=0.24, p<0.01)$. Univariate analysis comparing patients with normal lung function and RLD showed significantly more patients with increased BMI $(p<0.05)$ suffering of breathlessness $(p<$ $0.01)$, increased $\mathrm{HbAlc}(p<0.01)$, and increased triglycerides (online suppl. Table S1). Patients with a restrictive ventilation pattern showed no increased NT-proBNP compared to patients with normal lung function (93.1 \pm 11.6 vs. $92.3 \pm 17.6, p=$ nonsignificant). In patients with RLD, FVC, SB-DL ${ }_{\mathrm{CO}}$, and TLC-B were significantly decreased, while $\mathrm{FEV}_{1} / \mathrm{VC}$ showed significantly increased values (all $p<0.01$ ). Patients with long-term T2D had an independent risk in multivariate analysis (OR 5.82 [95\% CI 1.71-20.52], $p<0.01$ ) for RLD, while the risk of patients with newly diagnosed T2D showed a trend only towards RLD (OR 3.91 [95\% CI 0.9-17.1], $p=0.07$ ) (Fig. 2b). Prediabetes, triglycerides, and standard risk factors for RLD, such as age, sex, BMI, history of smoking, and CVD, were not significantly associated with an increased risk in this cohort (details are given in online suppl. Table S1).

\section{Correlation of Lung Function with Glucose} Metabolism and Diabetic Complications

Fasting glucose and $\mathrm{HbAlc}$ showed a significant negative association with FVC, SB-DL $\mathrm{CO}_{\mathrm{CO}}$ and TLC-B (online suppl. Fig. S3). Increased fasting glucose was significantly associated with decreased FVC $(r=-0.25, p<0.01$; online suppl. Fig. S3A), decreased SB-DL $\mathrm{CO}(r=-0.15, p<0.05$; online suppl. Fig. S3B), and decreased TLC-B $(r=-0.22$, $p<0.01$; online suppl. Fig. S3C). Similar associations were calculated for increased HbA1c with decreased FVC ( $r=$ $-0.28, p<0.01$; online suppl. Fig. S3D), decreased SB$\mathrm{DL}_{\mathrm{CO}}(r=-0.23, p<0.01$; online suppl. Fig. S3E), and decreased TLC-B ( $r=-0.22, p<0.01$; online suppl. Fig. $\mathrm{S} 3 \mathrm{~F})$. Correlation analyses were not able to show significant associations between $\mathrm{BMI}$ and $\mathrm{HbAlc}(r=0.14, p=$ $0.1)$ or between BMI and fasting glucose $(r=0.12, p=$ $0.15)$. Furthermore, in a multivariate linear regression, fasting glucose showed a significant negative association with FVC $(\beta=-0.21, T=-3.4, p<0.01)$ and with TLC-B $(\beta=-0.19, T=-3.0, p<0.01)$ after adjustment for age, sex, BMI, history of smoking, and patient group. In addition, $\mathrm{HbAlc}$ was independently negatively associated with FVC $(\beta=-0.24, T=-4.0, p<0.01)$, SB-DL $_{\mathrm{CO}}(\beta=$ $-0.18, T=-2.9, p<0.01)$, and TLC-B $(\beta=-0.19, T=-3.1$, $p<0.01$ ) (Table 2).

Additionally, multivariate logistic regression analysis was performed in patients with T2D to investigate independent associations and risk calculations for the predic- 
tive value of diabetes-related complications for the presence of RLD (online suppl. Table S2). Patients with T2D and RLD had significantly increased urinary albumincreatinine ratios $(p<0.05)$ with a significantly increased presence of nephropathy $(p<0.05)$, and a trend towards increased HbA1c $(p=0.08)$ (see online suppl. Table S2). Multivariate analysis in patients with T2D revealed that only presence of nephropathy was significantly associated with an increased risk of RLD (OR 2.73 [95\% CI 1.2$6.14], p<0.01$; online suppl. Fig. S4) compared to patients with T2D without nephropathy. However, presence of neuropathy and retinopathy showed no significant associations. Additional regression analysis showed an increased risk of RLD in patients with T2D with nephropathy (OR 8.57 [95\% CI 3.36-21.9], $p<0.01$ ) compared to nondiabetics (online suppl. Table S3). Exclusion of patients with nephropathy from the analysis revealed that patients with T2D without nephropathy still had an increased, nevertheless lower, risk of RLD (OR 2.66 [95\% CI 1.1-6.5], $p<0.05$ ] compared to nondiabetics (data not shown).

\section{Chest Computed Tomography, 6MWT, and Lung}

Tissue Staining

To assess whether pulmonary fibrosis was responsible for the observed RLD, 11 patients with breathlessness and restrictive lung pattern were offered MDCT and a 6MWT. Four of the 5 patients who agreed had interstitial lung abnormalities in the MDCT scans (Table 3; Fig. 3). Patient A showed only airway thickening but no signs of pulmonary fibrosis on MDCT scan (Fig. 3a) and normal FVC, normal TLC-B, and a normal 6MWT. Breathlessness was rated at 4 on the modified Borg scale (CR-10) after the 6MWT. Patients B and C showed subtle and mild intraand interlobular reticulations on MDCT scans (Fig. 3b, c) combined with clinical presence of nephropathy, slightly reduced walking distance (6MWT), and increased breathlessness on the Borg scale (Table 3). Patient D (a 74-yearold male) had a positive smoking history 20 years earlier, known polyneuropathy and nephropathy, but no known evidence for coronary heart disease. He had pronounced RLD, a reduced walking distance with increased rated breathlessness, and his MDCT scan revealed subpleural intralobular reticulations and ground-glass opacities (Table 3; Fig. 3d). Patient E had the most severe changes in lung function which correlated well with the MDCT findings (marked subpleural intralobular reticulation; Fig. 3e), decreased 6MWT distance $(340 \mathrm{~m} ; 71 \%)$, and severe breathlessness after activity (Borg scale 8) (Table $3)$.

Breathlessness and Restrictive Lung Disease
Table 2. Adjusted associations of glucose metabolism with lung function testing

\begin{tabular}{|c|c|c|c|c|c|c|}
\hline & \multicolumn{3}{|c|}{ Fasting glucose, $\mathrm{mg} / \mathrm{dL}$} & \multicolumn{3}{|c|}{$\mathrm{HbAlc}, \%(\mathrm{mmol} / \mathrm{mol})$} \\
\hline & $\beta$ & $T$ & $p$ & $\beta$ & $T$ & $p$ \\
\hline FVC, \% & -0.21 & -3.40 & $<0.01$ & -0.24 & -3.99 & $<0.01$ \\
\hline $\mathrm{SB}-\mathrm{DL}_{\mathrm{CO}}, \%$ & -0.11 & -1.79 & 0.07 & -0.18 & -2.88 & $<0.01$ \\
\hline TLC-B, \% & -0.19 & -3.0 & $<0.01$ & -0.19 & -3.11 & $<0.01$ \\
\hline
\end{tabular}

Data show results of the multivariable linear regression analyses (stepwise forward and backward) for FVC, SB-DL $\mathrm{DO}_{\mathrm{C}}$, and TLC$\mathrm{B}$ as dependent variables adjusted to age, sex, body mass index, arterial hypertension, cardiovascular disease, and patient group. FVC, forced vital capacity; $\mathrm{SB}-\mathrm{DL}_{\mathrm{CO}}$, single-breath diffusing capacity of the lung for carbon monoxide; TLC-B, total lung capacity measured with body plethysmography.

Normal lung tissue, which had been obtained from patients during surgery for lung cancer ( 3 with and 4 without diabetes) was studied for fibrosing lung disease. Morphological analysis showed increased fibrotic disease in patients with T2D compared to nondiabetics (Fig. 4a-h). Hematoxylin and eosin staining as well as trichrome staining of the tissue showed markedly increased alveolar septal fibrosis in patients with T2D compared to patients without diabetes.

\section{Discussion}

This study shows that increased breathlessness, RLD, and interstitial lung abnormalities may be associated with T2D. This is in contrast to international guidelines on idiopathic ILDs and IPF, were diabetes mellitus was not reported as an established risk factor for these diseases [23, 24]. Furthermore, pulmonary disorders were not listed as a diabetes-related complication by international diabetes associations $[27,28]$. In this study, the prevalence of RLD was between 20 - and $27 \%$ in patients with T2D. Furthermore, radiological and histological analyses suggest an association with fibrosing interstitial lung abnormalities. Prior studies support these findings by describing diabetes mellitus as a comorbidity and a risk factor for patients with IPF [13, 14]. Moreover, chronic breathlessness (measured by mMRC) was described as a negative prognostic indicator for survival in these patients [29].

The prevalence of moderate and severe chronic breathlessness was between $10-$ and $14 \%$ in patients with T2D in this study. Until now, no study exists which has inves- 


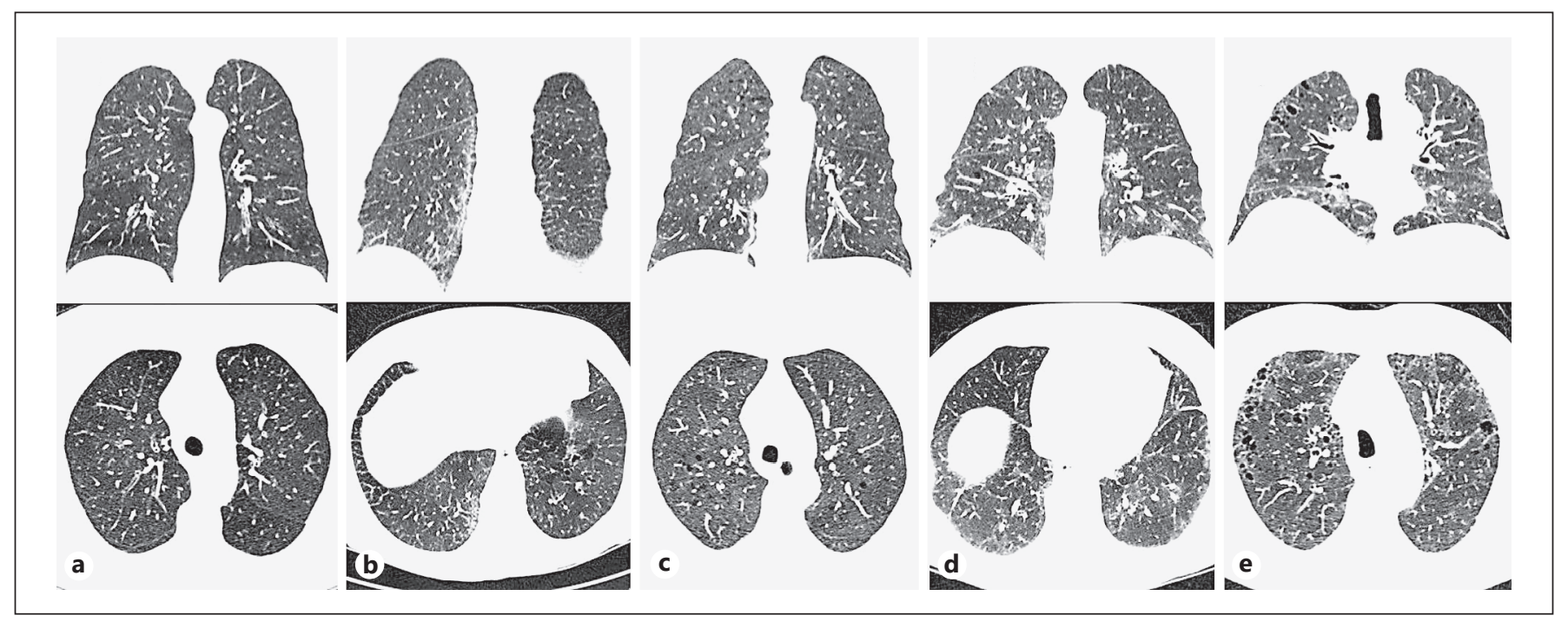

Fig. 3. MDCT chest scans in the coronary and axial plane from 5 patients with T2D. The clinical parameters of these patients are given separately in Table 2. a Airway wall thickening, but no signs of fibrosis. b Subtle subpleural intra- and interlobular reticulation. c Mild centrilobular emphysema and little subpleural intralobular reticulation. d Subpleural intralobular reticulation as well as patchy ground-glass opacities. e Marked subpleural intralobular reticulation as well as subpleural cysts and traction bronchiectasis. MDCT, multidetector computed tomography; T2D, type 2 diabetes.

Table 3. Clinical data of 5 patients who underwent MDCT and 6MWT

\begin{tabular}{llllll}
\hline & Patient A & Patient B & Patient C & Patient D & Patient E \\
\hline Age, years & 71 & 72 & 64 & 74 & 65 \\
Sex & female & male & female & male & male \\
Diabetes duration, years & 15 & 17 & 3 & 26 & 10 \\
History of smoking & no & no & no & yes & no \\
History of CVD & yes & no & no & no & no \\
HbA1c, \% (mmol/mol) & $7.3(56)$ & $7.1(54)$ & $6.1(43)$ & $8.2(66)$ & $8.1(65)$ \\
Nephropathy & no & yes & yes & yes & yes \\
Polyneuropathy & no & yes & no & yes & no \\
Retinopathy & no & no & no & no & no \\
FVC, \% & 82 & 98 & 78 & 70 & 64 \\
SB-DL CO, \% & 59 & 57 & 54 & 47 & 31 \\
TLC-B, \% & 78 & 79 & 79 & 74 & 70 \\
Fibrosing ILD (MCDT) & no & mild & mild & moderate & severe \\
6MWT distance, m & 400 & 490 & 380 & 358 & 340 \\
6MWT, \% of prediction & 98 & 98 & 91 & 74 & 71 \\
mBorg & 4 & 4 & 5 & 7 & 8 \\
\hline
\end{tabular}

Lung function parameters were given in percent of prediction. Results from MDCT were shown in Figure 3. 6MWT, Six-Minute Walk Test; CVD, cardiovascular disease; FVC, forced vital capacity; ILD, interstitial lung disease; mBorg, modified BORG scale for breathlessness (CR-10) after 6MWT; MDCT, multidetector computed tomography; $\mathrm{SB}-\mathrm{DL}_{\mathrm{CO}}$, single-breath diffusing capacity of the lung for carbon monoxide; TLC-B, total lung capacity measured with body plethysmography. 


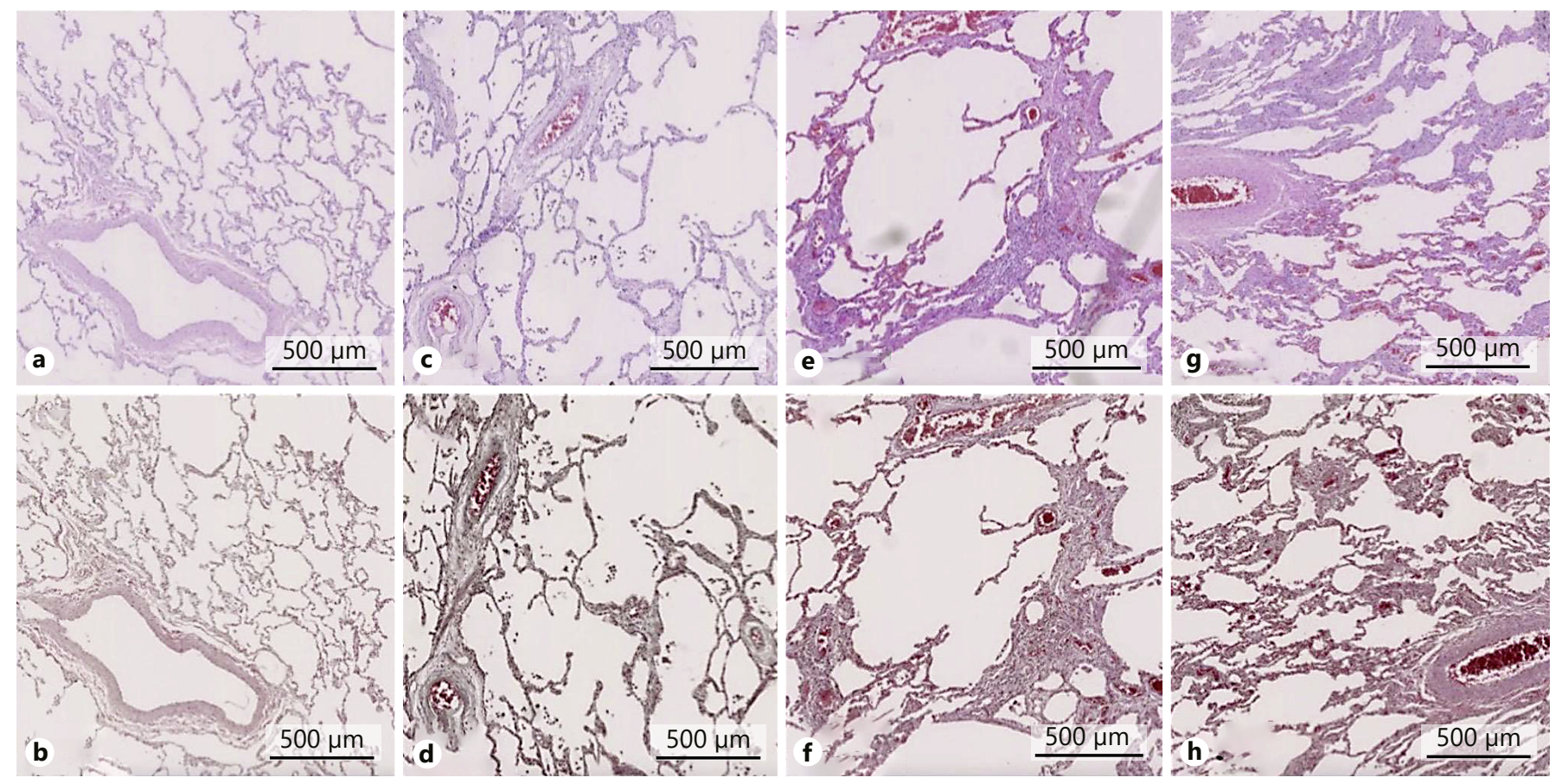

Fig. 4. Histopathology of lung tissues with $\mathrm{HE}$ and trichrome staining from 2 patients without diabetes (a-d) and 2 patients with T2D (e-h). Patients without diabetes showed normal alveolar structure on HE staining (a, c) and on trichrome staining $(\mathbf{b}, \mathbf{d})$. Patients with T2D showed increased interalveolar structure on HE staining $(\mathbf{e}, \mathbf{g})$ and increased positivity for trichrome staining in the interalveolar space $(\mathbf{f}, \mathbf{h})$. Pictures were made at $3.7 \times$ magnification with NDP.view2 (Hamamatsu Photonics Deutschland GmbH, Herrsching am Ammersee, Germany). $\mathrm{HE}$, hematoxylin and eosin; T2D, type 2 diabetes.

tigated the association of impaired lung function with breathlessness in patients with diabetes. In daily clinical practice, breathlessness or dyspnea are attributed to acute cardiovascular events, but only $11 \%$ of cases can be explained by CVDs $[1,2]$. One study reported that only $42.5 \%$ of patients with T2D and dyspnea had abnormal stress ECGs, and only $58.8 \%$ of these patients showed significant coronary stenosis [30]. Extrinsic factors like obesity, hypertension, and ventricular dysfunction could be possible reasons for breathlessness and pulmonary dysfunction [31]. The data from this current study did not show any evidence for extrinsically induced breathlessness or lung disorders; no history for chronic heart failure was reported, and breathlessness and RLD did not show any associations with BMI, NT-proBNP, or history of arterial hypertension. Thus, increased breathlessness and RLD may only be explained by an intrinsic factor, such as ILD.

The lung as a target organ for diabetes-related complications has been discussed in a few studies before [9-11, $32-35]$. Since spirometry was investigated in most pa- tients with T2D, a definitive differentiation between distinctive ventilation disorders was not possible [32-34]. Only a few studies have additionally included diffusing capacity as an outcome parameter and have therefore described a reduction in patients with diabetes [35]. The mean decreases of $10 \%$ for $\mathrm{FVC}$ and $\mathrm{SB}-\mathrm{DL}_{\mathrm{CO}}$ reported in this study are comparable to those in other studies [35]. Nevertheless, none of the patients in this study was found to have an obstructive lung disease. Van den Borst et al. [35] and Klein et al. [11] emphasized that the decrease in $\mathrm{FEV}_{1}$ was not as consistent as the reduction in FVC in patients with diabetes. A possible reason could be that patients with RLD typically showed increased $\mathrm{FEV}_{1} / \mathrm{VC}$, as found in this study $[23,24]$. Thus, obstructive lung disease is not a predominant problem in patients with T2D if high-risk patients (smokers and patients with asthma) are excluded.

The findings of this study were supported by random histological examination of lung tissue and MDCT scans in selected patients. Consistent with previous findings from autopsies, patients with T2D showed increased fi- 
brosing ILD [7]. Patients with IPF showed a prevalence of $25-32 \%$ for diabetes mellitus, and there is a risk of IPF in patients with diabetes, as shown retrospectively in casecontrol analyses $[13,14]$. In this study, the patterns seen on MDCT were different compared to the typical reticular and honeycomb appearance in IPF patients [12]. Four of 5 patients showed increased interstitial reticular lung abnormalities on MDCT scans, 1 patient showed additional emphysema, and 1 patient showed pulmonary cysts (Fig. 3). The significance of these MDCT scans is limited, but they suggest that ILD in patients with diabetes mellitus needs to be investigated more frequently. It remains to be determined whether this is related to a systemic effect on fibrosis formation in patients with diabetes. Pulmonary fibrosis has also been observed in experimental diabetes models [36-38]. Thus, diabetes itself may be associated with a generalized increased risk of fibrosis in several organs.

The pathomechanism of RLD and fibrosing ILD remains unclear. Previous studies in patients with and without diabetes have described associations between a restrictive lung pattern and glucose metabolism [32-34, 39]. These findings are supported by the results shown in this study (Table 3). However, the multivariate regression analysis was not able to confirm these associations for RLD (online suppl. Table S2). Thus, the association between glucose metabolism and RLD could be an observational effect, and future studies are required to clarify the definitive pathomechanisms. The regression analysis showed an independent association with presence of nephropathy only. The common hypothesis regarding pulmonary fibrosis involves oxidative stress, endothelial microinjuries, and platelet activation with consecutive inflammation [6]. Similar mechanisms have been described in diabetes-related complications, especially nephropathy $[5,6,40,41]$. Furthermore, low estimated glomerular filtration rate was associated with decreased survival in patients with IPF [42]. This indicates potentially common mechanisms for fibrosing ILD and established microvascular complications in patients with T2D.

\section{Technical Considerations and Limitations}

The cross-sectional design in a rather small cohort is the main limitation of this study. Larger studies with prospective endpoints are needed to investigate the natural cause of RLD and pulmonary fibrosis in patients with prediabetes and T2D as well as type 1 diabetes. Unfortunately, the small number of patients who consented to MDCT does not allow a clear assessment of ILD in patients with T2D. These MDCTs showed interstitial lung abnormalities in 4 out of 5 patients with significantly breathlessness and RLD, but further studies are needed to investigate possible specific changes in these patients. Furthermore, future studies should focus more on patients with diabetes mellitus and active smoking because smokers showed increased fibrosing interstitial lung abnormalities detected by chance [43]. Moreover, intervention studies are necessary to investigate possible new treatments in patients with diabetes.

\section{Conclusions}

Increased breathlessness, RLD, and interstitial lung abnormalities may be associated with T2D. Restrictive pulmonary function is related to glucose metabolism, and presence of nephropathy increases the risk of RLDs in patients with T2D. Due to these findings as well as results from animal studies and previous trials showing a significant association between restrictive lung disorders and diabetes mellitus, we conclude that pneumopathy is a late complication of diabetes and should be more frequently screened in patients with T2D, nephropathy, and breathlessness.

\section{Acknowledgments}

This study was supported by the Federal Ministry for Research (BMBF)/German Center for Diabetes Research (DZD e.V.; FKZ 82DZD00702), a DZD Grant 2016 (FKZ 82DZD0001G), and the Collaborative Research Center 1118 (CRC1118) by the Deutsche Forschungsgemeinschaft (DFG). The authors thank all participants in this study and Sandra Bischoff, Anita Pflästerer, Christa Kannengießer, and all other members of the study team.

\section{Statement of Ethics}

All participants involved in this study gave written informed consent. The study was approved by the local ethics committee of the University of Heidelberg (No. 515/2012, 232/2013, 245/2013, and 383/2016) and was performed in accordance with the 2013 version of the Declaration of Helsinki.

\section{Disclosure Statement}

Dr. Kopf reports grants from the Federal Ministry for Research (BMBF)/German Center for Diabetes Research (DZD e.V.) during the conduct of the study. Dr. Groener reports support from the Federal Ministry for Research (BMBF)/German Center for Diabetes Research (DZD e.V.) during the conduct of the study. Dr. Kender reports support from the Federal Ministry for Research (BMBF)/
Kopf et al. 
German Center for Diabetes Research (DZD e.V.) during the conduct of the study. Dr. Fleming reports support from the Federal Ministry for Research (BMBF)/German Center for Diabetes Research (DZD e.V.) and funding from the Deutsche Forschungsgemeinschaft (DFG) during the conduct of the study. C. Riedinger reports support from the Deutsche Forschungsgemeinschaft (DFG) during the conduct of the study. Dr. Volk reports support from the Deutsche Forschungsgemeinschaft (DFG) during the conduct of the study. Dr. Herpel reports support from the Deutsche Forschungsgemeinschaft (DFG) during the conduct of the study. Prof. Dr. Nawroth reports grants from the Federal Ministry for Research (BMBF)/German Center for Diabetes Research (DZD e.V.), from the Federal Ministry for Research (BMBF)/German Center for Diabetes Research (DZD e.V.), and from the Deutsche Forschungsgemeinschaft (DFG) during the conduct of the study. Dr. Brune, Dr. Wielpütz, Dr. Szendrödi, Dr. Pesta, Prof. Dr. Kauczor, Prof. Dr. Katus, and Prof. Dr. Kreuter have nothing to disclose. No conflicts of interest are reported with regard to this study.

\section{Author Contributions}

S. Kopf takes full responsibility for the work, including study design, access data, and the decision to submit and publish the manuscript. He was responsible for study design, patient recruitment, data collection, data analysis, and writing of the manuscript. J.B. Groener and Z. Kender were responsible for patient recruitment and data collection. T. Fleming contributed to writing and discussion of the manuscript. M. Brune and C. Riedinger were responsible for blood sample processing and analyses. N. Volk and E. Herpel were responsible for tissue collection, staining, and interpretation. D. Pesta and J. Szendrödi contributed to study design and manuscript discussion. M.O. Wielpütz and H.-U. Kauczor were responsible for MDCT scans and interpretation of results. H.A. Katus contributed to study design and manuscript discussion. M. Kreuter contributed to study design, data analysis, and manuscript discussion. P.P. Nawroth was responsible for study observation, study design, writing, and manuscript discussion.

\section{References}

1 Berliner D, Schneider N, Welte T, Bauersachs $\mathrm{J}$ : The differential diagnosis of dyspnea. Dtsch Arztebl Int 2016;113:834-845.

2 Frese T, Sobeck C, Herrmann K, Sandholzer $\mathrm{H}$ : Dyspnea as the reason for encounter in general practice. J Clin Med Res 2011;3:239246.

- 3 Kornum JB, Christensen S, Grijota M, Pedersen L, Wogelius P, Beiderbeck A, Sørensen HT: The incidence of interstitial lung disease 1995-2005: a Danish nationwide populationbased study. BMC Pulm Med 2008;8:24.

-4 Maher TM, Wells AU, Laurent GJ: Idiopathic pulmonary fibrosis: multiple causes and multiple mechanisms? Eur Respir J 2007;30:835839.

5 Richeldi L, Collard HR, Jones MG: Idiopathic pulmonary fibrosis. Lancet 2017;389:19411952.

-6 Yan Z, Kui Z, Ping Z: Reviews and prospectives of signaling pathway analysis in idiopathic pulmonary fibrosis. Autoimmun Rev 2014;13:1020-1025.

7 Sandler M: Is the lung a "target organ" in diabetes mellitus? Arch Intern Med 1990;150: 1385-1388.

8 Hyldgaard C, Hilberg O, Muller A, Bendstrup E: A cohort study of interstitial lung diseases in central Denmark. Respir Med 2014;108: 793-799.

-9 Benbassat CA, Stern E, Kramer M, Lebzelter J, Blum I, Fink G: Pulmonary function in patients with diabetes mellitus. Am J Med Sci 2001;322:127-132.

10 Yang J, Xue Q, Miao L, Cai L: Pulmonary fibrosis: a possible diabetic complication. Diabetes Metab Res Rev 2011;27:311-317.

11 Klein OL, Krishnan JA, Glick S, Smith LJ: Systematic review of the association between lung function and type 2 diabetes mellitus. Diabet Med 2010;27:977-987.
12 Kim YJ, Park JW, Kyung SY, Lee SP, Chung MP, Kim YH, Lee JH, Kim YC, Ryu JS, Lee HL, Park CS, Uh ST, Lee YC, Kim KH, Chun YJ, Park YB, Kim DS, Jegal Y, Lee JH, Park MS, Jeong SH: Clinical characteristics of idiopathic pulmonary fibrosis patients with diabetes mellitus: the national survey in Korea from 2003 to 2007. J Korean Med Sci 2012;27:756-760.

13 Kreuter M, Ehlers-Tenenbaum S, Palmowski K, Bruhwyler J, Oltmanns U, Muley T, Heussel CP, Warth A, Kolb M, Herth FJ: Impact of comorbidities on mortality in patients with idiopathic pulmonary fibrosis. PLoS One 2016;11:e0151425.

14 Enomoto T, Usuki J, Azuma A, Nakagawa T, Kudoh S: Diabetes mellitus may increase risk for idiopathic pulmonary fibrosis. Chest 2003; 123:2007-2011.

15 Levey AS, Stevens LA, Schmid CH, Zhang YL, Castro AF 3rd, Feldman HI, Kusek JW, Eggers P, Van Lente F, Greene T, Coresh J; CKDEPI (Chronic Kidney Disease Epidemiology Collaboration): A new equation to estimate glomerular filtration rate. Ann Intern Med 2009;150:604-612.

16 Kishaba T, Nagano H, Nei Y, Yamashiro S: Clinical characteristics of idiopathic pulmonary fibrosis patients according to their smoking status. J Thorac Dis 2016;8:1112-1120.

17 Quanjer PH, Stanojevic S, Cole TJ, Baur X, Hall GL, Culver BH, Enright PL, Hankinson JL, Ip MS, Zheng J, Stocks J; ERS Global Lung Function Initiative: Multi-ethnic reference values for spirometry for the 3-95-yr age range: the global lung function 2012 equations. Eur Respir J 2012;40:1324-1343.

18 Robinson PD, Latzin P, Verbanck S, Hall GL, Horsley A, Gappa M, Thamrin C, Arets HG, Aurora P, Fuchs SI, King GG, Lum S, Macleod K, Paiva M, Pillow JJ, Ranganathan S, Ratjen F, Singer F, Sonnappa S, Stocks J, Subbarao P,
Thompson BR, Gustafsson PM: Consensus statement for inert gas washout measurement using multiple- and single-breath tests. Eur Respir J 2013;41:507-522.

19 Pellegrino R, Viegi G, Brusasco V, Crapo RO, Burgos F, Casaburi R, Coates A, van der Grinten CP, Gustafsson P, Hankinson J, Jensen R, Johnson DC, MacIntyre N, McKay R, Miller MR, Navajas D, Pedersen OF, Wanger $\mathrm{J}$ : Interpretative strategies for lung function tests. Eur Respir J 2005;26:948-968.

20 Brack T, Jubran A, Tobin MJ: Dyspnea and decreased variability of breathing in patients with restrictive lung disease. Am J Respir Crit Care Med 2002;165:1260-1264.

21 Kopf S, Oikonomou D, Zdunek D, von Eynatten M, Hess G, Nawroth PP, Bierhaus A, Humpert PM: Urinary n-acetyl-beta-d-glucosaminidase excretion: an indicator of neuropathy in type 2 diabetes. Exp Clin Endocrinol Diabetes 2013;121:601-606.

22 Young MJ, Boulton AJ, MacLeod AF, Williams DR, Sonksen PH: A multicentre study of the prevalence of diabetic peripheral neuropathy in the United Kingdom hospital clinic population. Diabetologia 1993;36:150-154.

23 Raghu G, Collard HR, Egan JJ, Martinez FJ, Behr J, Brown KK, Colby TV, Cordier JF, Flaherty KR, Lasky JA, Lynch DA, Ryu JH, Swigris JJ, Wells AU, Ancochea J, Bouros D, Carvalho C, Costabel U, Ebina M, Hansell DM, Johkoh T, Kim DS, King TE Jr, Kondoh Y, Myers J, Müller NL, Nicholson AG, Richeldi L, Selman M, Dudden RF, Griss BS, Protzko SL, Schünemann HJ; ATS/ERS/JRS/ALAT Committee on Idiopathic Pulmonary Fibrosis: An official ATS/ERS/JRS/ALAT statement: idiopathic pulmonary fibrosis: evidence-based guidelines for diagnosis and management. Am J Respir Crit Care Med 2011;183:788-824.
Breathlessness and Restrictive Lung Disease 
-24 Travis WD, Costabel U, Hansell DM, King TE Jr, Lynch DA, Nicholson AG, Ryerson CJ, Ryu $\mathrm{JH}$, Selman M, Wells AU, Behr J, Bouros D, Brown KK, Colby TV, Collard HR, Cordeiro CR, Cottin V, Crestani B, Drent M, Dudden RF, Egan J, Flaherty K, Hogaboam C, Inoue Y, Johkoh T, Kim DS, Kitaichi M, Loyd J, Martinez FJ, Myers J, Protzko S, Raghu G, Richeldi L, Sverzellati N, Swigris J, Valeyre D; ATS/ ERS Committee on Idiopathic Interstitial Pneumonias: An official American Thoracic Society/European Respiratory Society statement: update of the international multidisciplinary classification of the idiopathic interstitial pneumonias. Am J Respir Crit Care Med 2013;188:733-748.

25 Enright PL, Sherrill DL: Reference equations for the six-minute walk in healthy adults. Am J Respir Crit Care Med 1998;158:1384-1387.

26 Wilson RC, Jones PW: A comparison of the visual analogue scale and modified Borg scale for the measurement of dyspnoea during exercise. Clin Sci (Lond) 1989;76:277-282.

$\checkmark 27$ American Diabetes Association: 10. Microvascular complications and foot care. Diabetes Care 2017;40(suppl 1):S88-S98.

28 IDF Clinical Guidelines Task Force: Global Guideline for Type 2 Diabetes: recommendations for standard, comprehensive, and minimal care. Diabet Med 2006;23:579-593.

-29 Nishiyama O, Taniguchi H, Kondoh Y, Kimura T, Kato K, Kataoka K, Ogawa T, Watanabe F, Arizono S: A simple assessment of dyspnoea as a prognostic indicator in idiopathic pulmonary fibrosis. Eur Respir J 2010; 36:1067-1072.
30 Kim MK, Baek KH, Song KH, Kwon HS, Lee JM, Kang MI, Yoon KH, Cha BY, Son HY, Lee KW: Exercise treadmill test in detecting asymptomatic coronary artery disease in type 2 diabetes mellitus. Diabetes Metab J 2011;35: 34-40.

31 Scarlata S, Costanzo L, Giua R, Pedone C, Incalzi RA: Diagnosis and prognostic value of restrictive ventilatory disorders in the elderly: a systematic review of the literature. Exp Gerontol 2012;47:281-289.

32 Davis WA, Knuiman M, Kendall P, Grange V, Davis TM; Fremantle Diabetes Study: Glycemic exposure is associated with reduced pulmonary function in type 2 diabetes: the Fremantle Diabetes Study. Diabetes Care 2004; 27:752-757.

33 Davis TM, Knuiman M, Kendall P, Vu H, Davis WA: Reduced pulmonary function and its associations in type 2 diabetes: the Fremantle Diabetes Study. Diabetes Res Clin Pract 2000; 50:153-159.

34 Teeter JG, Riese RJ: Cross-sectional and prospective study of lung function in adults with type 2 diabetes: the Atherosclerosis Risk in Communities (ARIC) study: response to Yeh et al. Diabetes Care 2008;31:e82.

35 Van den Borst B, Gosker HR, Zeegers MP, Schols AM: Pulmonary function in diabetes: a metaanalysis. Chest 2010;138:393-406.

6 Papinska AM, Soto M, Meeks CJ, Rodgers KE: Long-term administration of angiotensin (17) prevents heart and lung dysfunction in a mouse model of type 2 diabetes $(\mathrm{db} / \mathrm{db})$ by reducing oxidative stress, inflammation and pathological remodeling. Pharmacol Res 2016;107:372-380.
$37 \mathrm{Hu}$ Y, Ma Z, Guo Z, Zhao F, Wang Y, Cai L, Yang J: Type 1 diabetes mellitus is an independent risk factor for pulmonary fibrosis. Cell Biochem Biophys 2014;70:1385-1391.

- 38 Kumar V, Fleming T, Terjung S, Gorzelanny C, Gebhardt C, Agrawal R, Mall MA, Ranzinger J, Zeier M, Madhusudhan T, Ranjan S, Isermann $\mathrm{B}$, Liesz $\mathrm{A}$, Deshpande $\mathrm{D}$, Haring HU, Biswas SK, Reynolds PR, Hammes HP, Peperkok R, Angel P, Herzig S, Nawroth PP: Homeostatic nuclear RAGE-ATM interaction is essential for efficient DNA repair. Nucleic Acids Res 2017;45:10595-10613.

39 Oh IH, Park JH, Lee CH, Park JS: The association of normal range glycated hemoglobin with restrictive lung pattern in the general population. PLoS One 2015; 10:e0117725.

40 Jagadapillai R, Rane MJ, Lin X, Roberts AM, Hoyle GW, Cai L, Gozal E: Diabetic microvascular disease and pulmonary fibrosis: the contribution of platelets and systemic inflammation. Int J Mol Sci 2016;17:E1853.

-41 King TE Jr, Pardo A, Selman M: Idiopathic pulmonary fibrosis. Lancet 2011;378:19491961.

42 Ikezoe K, Handa T, Tanizawa K, Yokoi H, Kubo T, Aihara K, Sokai A, Nakatsuka Y, Hashimoto S, Uemasu K, Sato S, Muro S, Nagai S, Yanagita M, Chin K, Hirai T, Taguchi Y, Mishima M: Chronic kidney disease predicts survival in patients with idiopathic pulmonary fibrosis. Respiration 2017;94: 346-354.

-43 Chung JH, Richards JC, Koelsch TL, MacMahon H, Lynch DA: Screening for lung cancer: incidental pulmonary parenchymal findings. AJR Am J Roentgenol 2018;210:503-513. 